\title{
Synchronized Dual-Polarity Electrospray Ionization Mass Spectrometry
}

\author{
Han-Kwang Chen, ${ }^{a}$ Chia-Kai Chang, ${ }^{b}$ Chih-Che $\mathrm{Wu}^{\mathrm{a}}$ \\ Ming-Chia Huang, ${ }^{b}$ and Yi-Sheng Wang ${ }^{b}$ \\ ${ }^{a}$ Department of Chemistry, Chi Nan University, Nantou, Taiwan, Republic of China \\ ${ }^{\mathrm{b}}$ Genomics Research Center, Academia Sinica, Taipei, Taiwan, Republic of China
}

\begin{abstract}
This work describes the synchronized dual-polarity (DP) electrospray ionization (ESI) method and demonstrates the first DP ESI mass spectra obtained using two mass spectrometers. Stable double Taylor cones were produced by applying two counter electric voltages with opposite polarities to one electrosprayer. The development of double Taylor cones required higher extraction voltages than conventional ESI, but DP ESI worked effectively at liquid flow rate range three times wider than conventional ESI. Using pure methanol, the emission currents of the two cones were neutralized and no current was drawn from the sprayer. Synchronized DP mass spectra were obtained using electrospray calibrants dissolved in methanol solution of low water content. For bovine insulin with conventional electrospray solution, the gas-assisted electrospray delivered satisfactory sensitivity and stability for routine mass analyses. (J Am Soc Mass Spectrom 2009, 20, 2254-2257) (c) 2009 American Society for Mass Spectrometry
\end{abstract}

$\mathrm{E}$ lectrospray ionization (ESI) is an indispensable ion production method [1], but its polarity selection nature limits the ions it can produce to one charge polarity at a time. Such an effect is inevitable in conventional ESI because only one extraction electrode is involved; the ions disfavoring the extraction field are retained in the solution, and are undetectable. Polarity switching [2] is an approach to monitor positive and negative ions alternatively by a mass spectrometer. It is utilized with liquid-chromatographic systems to identify protein modifications [3,4], metabolites [5], and environmental hazards [6], but the instrument performance declines due to duty cycle problems, and the few seconds required for signal recovery after every such switch.

The best strategy to obtain positive and negative mass spectra is to employ contemporaneous dual-polarity (DP) ion detection $[7,8]$. The only DP ionization approach involving liquid samples is the field-induced droplet ionization (FIDI) [9], in which positive and negative liquid jets are produced from falling droplets within a strong d.c. electric field. FIDI can be applied to droplets suspended at a capillary using pulsed extraction fields [10], but pulsed ion extraction is unsuitable for flowing liquids, especially for liquid-chromatography, because reproducing extraction conditions and recording rapidly changing spectra are difficult. To our knowledge, syn-

Address reprint requests to Dr. Y.-S. Wang, Genomics Research Center, Academia Sinica, 128, Academia Road, Section 2, Nankang District, Taipei 115, Taiwan, R. O. C. E-mail: wer@gate.sinica.edu.tw chronized DP mass spectrometry (MS) of liquid samples has never been reported in the past.

This work demonstrates a synchronized DP ESI method with continuous sample injection. The electrosprayer was installed between two extraction electrodes with opposite d.c. voltages. The resultant DP electrospray (ES) properties were studied in relation to the magnitudes of extraction voltages and liquid flow rates. Synchronized DP mass analysis was conducted using two ESI-ion trap mass spectrometers. The method is advantageous for the development of novel highthroughput DP MS.

\section{Experimental}

The ES properties were studied using an imaging system. The electrosprayer was a $150 \mathrm{~mm}$ stainless steel tube with an o.d. of $1.07 \mathrm{~mm}$ and an i.d. of $0.77 \mathrm{~mm}$. It was installed at the center of the $12 \mathrm{~mm}$ space developed by two parallel extraction electrodes (top panel, Figure 1). A microscope (SZ61; Olympus, Tokyo, Japan) equipped with a charged-coupled device (CCD) (DFWX710; Sony UK, Brooklands, UK) was used to image the Taylor cones with back-illumination. Exposure time of the CCD was $40 \mu \mathrm{s}$ and frame rate was $7.5 \mathrm{fps}$. The electrosprayer was grounded through a $5.3 \mathrm{M} \Omega$ resistor and the ES current departing from the sprayer was monitored using an oscilloscope that measured the voltage difference across the resistor. Spraying liquid was infused out of the electrosprayer tip through a fused silica capillary (FS-115; Upchurch Scientific, Oak 

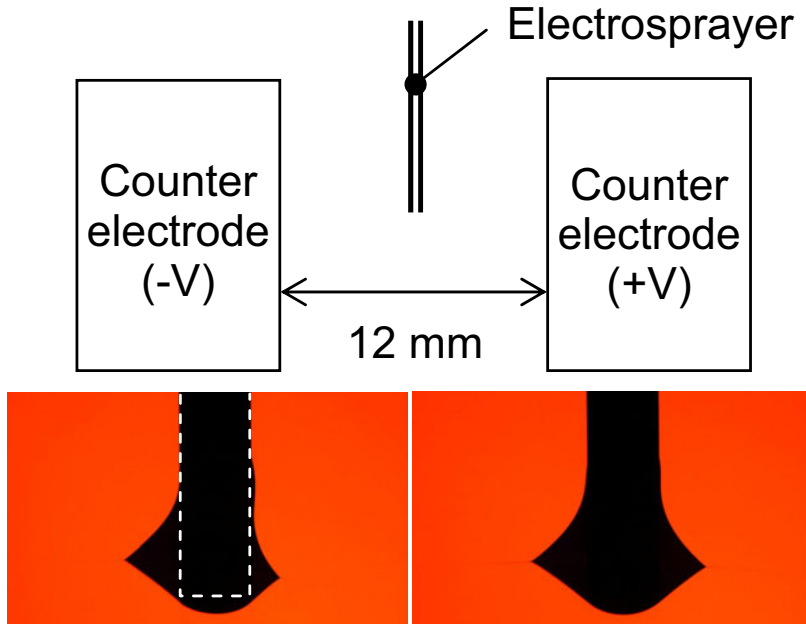

(a)

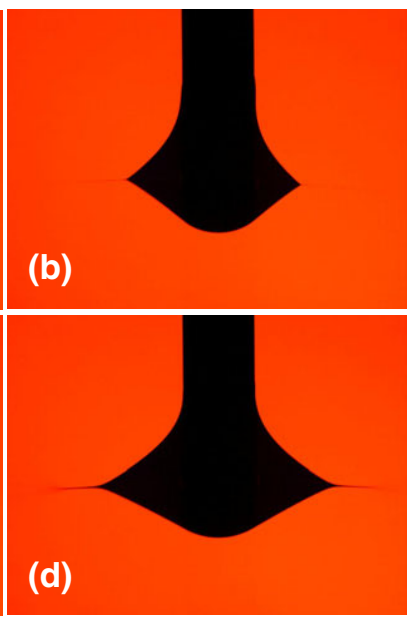

(c)

Figure 1. Imaging system and DP Taylor cones obtained with methanol flow rates of (a) 5, (b) 20, (c) 70, and (d) $150 \mu \mathrm{L} / \mathrm{min}$. The dashed line in (a) represents the electrosprayer.

Harbor, WA, USA). The d.c. extraction voltage was supplied to the electrode in series through a currentlimiting $80 \mathrm{M} \Omega$ resistor and a battery-powered potential meter. The ES currents deposited onto the electrodes were estimated by dividing the voltage reading by the internal resistance $(10 \mathrm{M} \Omega)$ of the meter.

Mass analyses were conducted using a Bruker Esquire 3000 plus (Bruker Daltonik, Bremen, Germany) and an Esquire 6000 plus in positive and negative ion mode, respectively. The removable source covers of the instruments were removed to position the two instruments coaxially opposite each other. An extension electrode was installed on the entrance capillary to develop an $8-10 \mathrm{~mm}$ space between the two instruments. Currentlimiting resistors $(80 \mathrm{M} \Omega)$ were installed to the extraction electrodes to protect the electronics. The electrosprayer used in the imaging experiment was secured at the center of the space to align the apices of the Taylor cones with the entrance capillaries of the instruments. The liquid flow rate was $10 \mu \mathrm{L} / \mathrm{min}$ and the extraction voltages of the two instruments were +4800 and -4800 $\mathrm{V}$. When the gas-assisted electrosprayer of the instrument was used, the original entrance caps were retained with a space of $49 \mathrm{~mm}$ between the two instruments. This sprayer was secured $17 \mathrm{~mm}$ above the entrance axis, and its horizontal position was adjusted to optimize the signal intensity. In gas-assisted ESI, the liquid flow rate was $2.5 \mu \mathrm{L} / \mathrm{min}$ with a nebulizing nitrogen pressure of $24 \mathrm{psi}$, and the extraction voltages were
+6000 and $-6000 \mathrm{~V}$. The dry gas temperature and flow rate in all measurements were $200{ }^{\circ} \mathrm{C}$ and $10-12 \mathrm{~L} / \mathrm{min}$, respectively. The mass spectra were the average of data of 1 to $2 \mathrm{~min}$.

Pure methanol (Sigma-Aldrich, St. Louis, MO, USA) was used as the spray liquid in imaging experiments for its low surface tension and high electric conductivity. The spray solution for mass analysis without nebulizing gas was a standard ES calibrant solution (G2421A; Agilent Technologies, Santa Clara, CA, USA) that contains various organic and inorganic chemicals in 95\% aqueous acetonitrile. It was diluted 20 times with methanol before use. The spray solution for gas-assisted ESI was bovine insulin (15500; Sigma-Aldrich) with a concentration of $1 \mathrm{pmol} / \mu \mathrm{L}$ in $50 \%$ aqueous methanol. The insulin solution was adjusted to $\mathrm{pH} 3$ with formic acid.

\section{Results and Discussion}

\section{Characteristics of Dual-Polarity Taylor Cones}

Two stable Taylor cones presented simultaneously at the electrosprayer tip when suitable extraction voltages were used (Figure 1a-d). The positively-charged cone developed toward the negatively-biased electrode and negative cone toward the positive electrode. A difference in magnitude of $>4 \%$ between the two extraction voltages made the cone of the low magnitude side diminish. The existence of stable double Taylor cones indicates that continuous and simultaneous emission of positive and negative ions from a single electrosprayer is feasible. This is in contrast to the generally-held concept that an ESI must be subject to polarity-selection effects.

The cone shapes elongated as flow rate increased and the two sides had roughly the same dimensions when $\geq 10 \mu \mathrm{L} / \mathrm{min}$ (Figure $1 \mathrm{~b}-\mathrm{d}$ ), but the negative cone had a smaller dimension than the positive cone when $<10 \mu \mathrm{L} /$ min (Figure 1a). When $<5 \mu \mathrm{L} / \mathrm{min}$, the pulsation of one cone induced severe droplet oscillations and the ES emission rapidly ceased. The unique spray characteristics of DP ESI were also shown in the critical extraction voltage and the effective liquid flow rate range.

Extraction voltages. DP ESI requires higher operational voltages than those used for conventional ESI. Figure $2 \mathrm{a}$ displays the appearances of Taylor cones and emission currents of methanol as a function of extraction voltage with a flow rate of $10 \mu \mathrm{L} / \mathrm{min}$. The critical voltage to produce double Taylor cones was approximately \pm 7 $\mathrm{kV}$, whereas the production of a single Taylor cone in conventional ESI (i.e., keeping one extraction electrode grounded) required roughly $4 \mathrm{kV}$ in either polarity. Because the theoretical Taylor limit [11] depends only on droplet diameter when the spray liquid is the same, the $75 \%$ higher extraction voltage used for DP ESI than conventional ESI is due to the decline of the effective electric field near the sprayer when counter extraction 

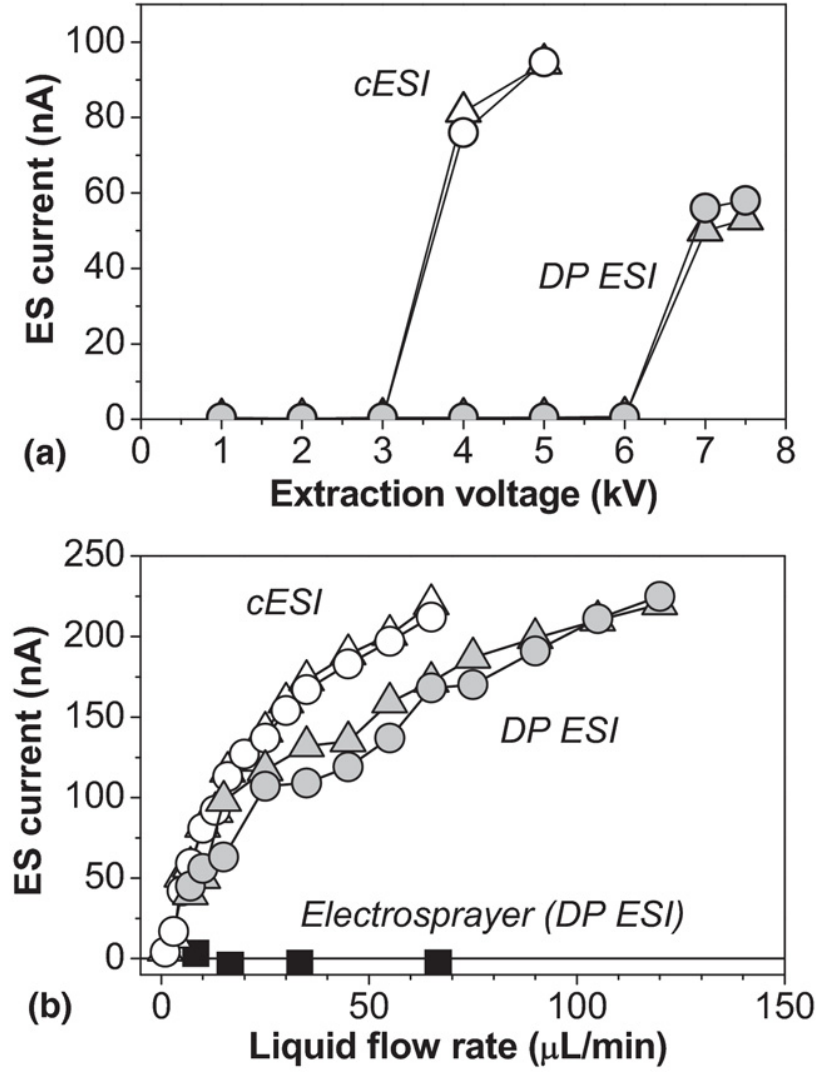

Figure 2. Methanol spray characteristics versus extraction voltage (a) and liquid flow rate (b). With a flow rate of $10 \mu \mathrm{L} / \mathrm{min}$, the onset voltage of ES emission in DP and conventional ESI (cESI) were $\pm 7 \mathrm{kV}$ and $\pm 4 \mathrm{kV}$, respectively (a). With appropriate extraction voltages, the DP ESI worked stably in $5-120 \mu \mathrm{L} / \mathrm{min}$, whereas the burst mode of conventional ESI started at $65 \mu \mathrm{L} / \mathrm{min}$ (b). Black squares represent currents departing from DP electrosprayer. Triangles represent positive ions and circles represent negative ions. Filled and open symbols represent the data of DP and conventional ESI, respectively.

voltages present, as was verified by an electro-optical simulation (data not shown).

The ES currents received by the negative and positive electrodes at the critical voltage of DP mode were 50 and $56 \mathrm{nA}$, respectively; the corresponding value observed in conventional ESI mode were 82 and $76 \mathrm{nA}$, respectively. The lower current on each side in DP mode than in conventional ESI is presumably due to splitting of the liquid into positive and negative streams. As a consequence, the Taylor cone on one side sprayed only half of the liquid, compared with conventional ESI at the same flow rate.

Liquid flow rates. With sufficient extraction voltages the double cone shapes remained stable at 5-200 $\mu \mathrm{L} /$ min (Figure $2 b$ ). In conventional ESI, the burst mode started from $65 \mu \mathrm{L} / \mathrm{min}$ and neither stable cones nor stable ES current were obtained. The emission currents in DP ESI increased with increases of the methanol flow rate until the current oscillated seriously above 120 $\mu \mathrm{L} / \mathrm{min}$. Such oscillation is possibly due to the field emission between the two electrodes when large amounts of liquid accumulate on the high-voltage electrodes [12]. Disregarding the current oscillation, DP ESI accepts liquid flow rate range three times wider than conventional ESI.

Interestingly, no net electric current was provided by the electrosprayer. The two extraction electrodes received ES currents of similar magnitudes but opposite polarities; the two streams were presumably neutralized and no current was drawn from the electrosprayer. This spraying characteristic also differed from conventional ESI, in which emission currents always flow from sprayer to counter electrode, making counter ions flow backward toward the capillary [13, 14]. In DP ESI, the counter ions in a Taylor cone may migrate toward another cone; most ions are extracted away from the sprayer by the side with attractive field. Thus, the effects of backward migration of ions and the resultant electrochemical reactions inside the capillary are likely suppressed in DP ESI.

\section{Dual-Polarity Mass Spectrometry}

The ES calibrant solution produced double Taylor cones between the two mass spectrometers, similar to the observation in imaging experiments. The double cones switched sharply to a single cone when the effective potential changed or the liquid flow rate fluctuated. When the apices of the double Taylor cones aligned with the entrance orifices, stable DP mass spectra were obtained (Figure 3a). The spectral features were the same as those obtained by using conventional ESI, suggesting the ions were produced by similar mechanism as conventional ESI. The only noticeable difference was the higher contaminant signal present in DP ESI, especially those at $\mathrm{m} / \mathrm{z}$ near 1306 and 1807 . However, liquids with high surface tensions, such as a methanol solution containing $10 \%$ water, possess a higher Taylor limit and result in unstable sprays. Such solutions are commonly used to dissolve biological samples, but serious discharge occurred because high extraction voltages were used.

The use of nebulizing gas reduced the extraction voltage and stabilized the spray [15]. Figure $3 \mathrm{~b}$ shows the DP mass spectra of insulin with the conventional ES solution obtained with gas-assisted ESI. The positive ions had a charge distribution of $3-6$, whereas the negative spectrum had an intense -3 state and a weak -4 state. These spectral patterns also agreed with those obtained using conventional gas-assisted ESI, but the best spray position was with the sprayer offset toward the negative instrument. One drawback of using DP ESI was the necessary increase in ion accumulation time from 1-5 ms to $10-50 \mathrm{~ms}$, but such accumulation times were still significantly faster than the speed of polarity switching. 

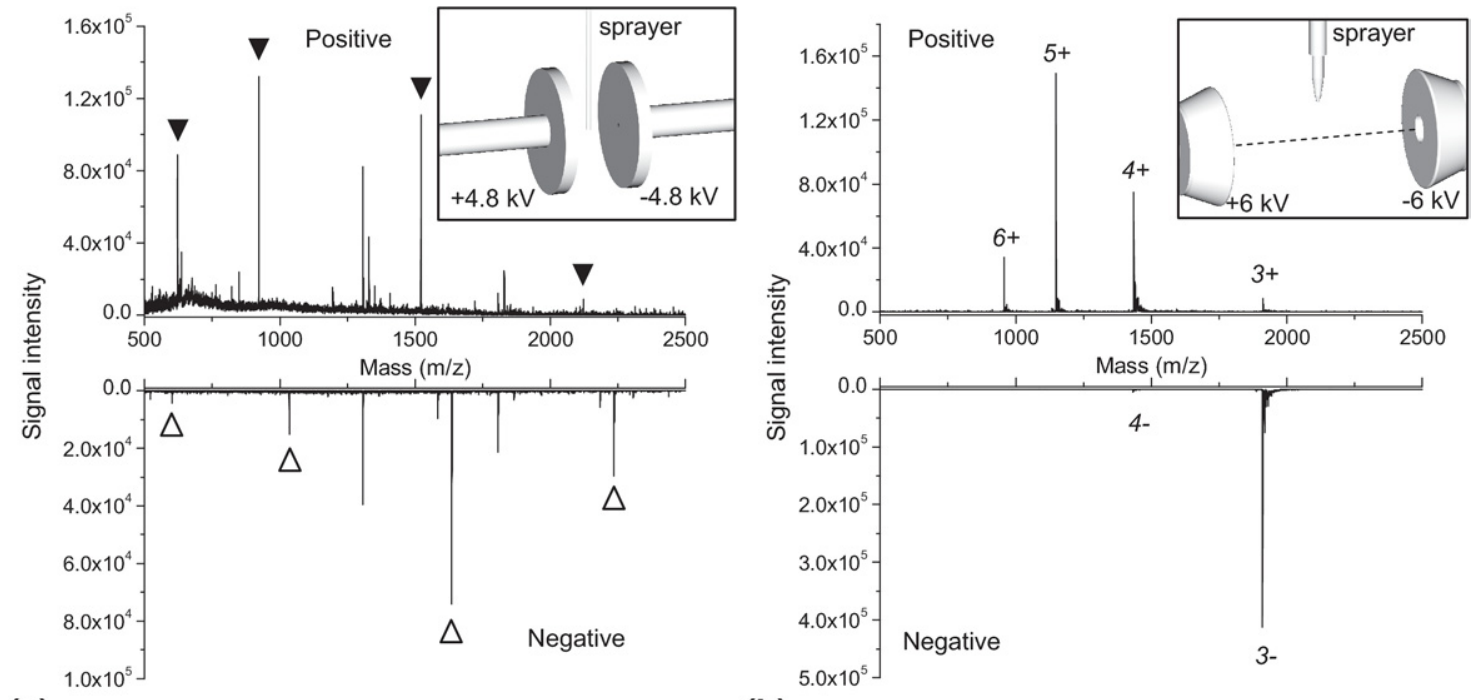

(a)

(b)

Figure 3. Synchronized DP mass spectra of ES calibrants obtained using a tube electrosprayer (a) and insulin using a gas-assisted electrosprayer (b). Filled and open symbols in (a) represent the features of ES calibrants. Insets show the source configuration of each experiment.

\section{Conclusions}

Synchronized DP ESI is effective by using two extraction voltages, as evidenced by the formation of double Taylor cones and the obtained DP mass spectra. This method is superior to the polarity switching for its speed and stability. Although DP ESI required higher extraction voltages than conventional ESI, it is suitable for wider liquid flow rate range. The limitations of DP ESI are the necessity for low surface tension solutions and high liquid flow rate, but the unsatisfactory performance when using high water-containing solutions was solved by utilizing gas-assisted electrospray.

\section{Acknowledgments}

The authors acknowledge support for this work by Academia Sinica and the National Science Council of Taiwan (contract no. NSC 96-2113-M-001-027-MY2).

\section{References}

1. Fenn, J. B.; Mann, M.; Meng, C. K.; Wong, S. F.; Whitehouse, C. M. Electrospray Ionization for Mass-Spectrometry of Large Biomolecules. Science 1989, 246, 64-71.

2. Hunt, D. F.; Stafford, G. C.; Crow, F. W.; Russell, J. W. Pulsed Positive Negative-Ion Chemical Ionization Mass-Spectrometry. Anal. Chem. 1976, 48, 2098-2105.

3. Annan, R.; Huddleston, M.; Verma, R.; Deshaies, R.; Carr, S. A Multidimensional Electrospray MS-Based Approach to Phosphopeptide Mapping. Anal. Chem. 2001, 73, 393-404.

4. Williamson, B. L.; Marchese, J.; Morrice, N. A. Automated Identification and Quantification of Protein Phosphorylation Sites by LC/MS on a
Hybrid Triple Quadrupole Linear Ion Trap Mass Spectrometer. Mol. Cell. Proteom. 2006, 5, 337-346.

5. Bu, H. Z.; Magis, L.; Knuth, K.; Eitelbaum, P. High-Throughput Cytochrome P450 (CYP) Inhibition Screening via Cassette Probe-Dosing Strategy. I. Development of Direct Injection/On-Line Guard Cartridge Extraction/Tandem Mass Spectrometry for the Simultaneous Detection of CYP Probe Substrates and their Metabolites. Rapid Commun. Mass Spectrom. 2000, 14, 1619-1624.

6. Leandro, C.; Hancock, P.; Fussell, R.; Keely, B. Ultra-Performance Liquid Chromatography for the Determination of Pesticide Residues in Foods by Tandem Quadrupole Mass Spectrometry with Polarity Switching. J. Chromatogr. A 2007, 1144, 161-169.

7. Hinz, K. P.; Kaufmann, R.; Spengler, B. Simultaneous Detection of Positive and Negative Ions from Single Airborne Particles by RealTime Laser Mass Spectrometry. Aerosol. Sci. Technol. 1996, 24, 233 242.

8. Tsai, S. T.; Chen, C. W.; Huang, L. C. L.; Huang, M. C.; Chen, C. H.; Wang, Y. S. Simultaneous Mass Analysis of Positive and Negative Ions Using a Dual-Polarity Time-of-Flight Mass Spectrometer. Anal. Chem. 2006, 78, 7729-7734.

9. Grimm, R. L.; Beauchamp, J. L. Field-Induced Droplet Ionization Mass Spectrometry. J. Phys. Chem. B 2003, 107, 14161-14163.

10. Grimm, R. L.; Hodyss, R.; Beauchamp, J. L. Probing Interfacial Chemistry of Single Droplets with Field-Induced Droplet Ionization Mass Spectrometry: Physical Adsorption of Polycyclic Aromatic Hydrocarbons and Ozonolysis of Oleic Acid and Related Compounds. Anal. Chem. 2006, 78, 3800-3806.

11. Taylor, G. Disintegration of Water Drops in Electric Field. Proc. R. Soc. London Ser. A Math. Phys. Eng. Sci. 1964, 280, 383-397.

12. Macky, W. A. Some Investigations on the Deformation and Breaking of Water Drops in Strong Electric Fields. Proc. R. Soc. London Ser. A Cont. Pap. Math. Phys. Char. 1931, 133, 565-587.

13. Blades, A. T.; Ikonomou, M. G.; Kebarle, P. Mechanism of Electrospray Mass-Spectrometry-Electrospray as an Electrolysis Cell. Anal. Chem. 1991, 63, 2109-2114.

14. Van Berkel, G. J. Electrolytic Deposition of Metals on to the HighVoltage Contact in an Electrospray Emitter: Implications for Gas-Phase Ion Formation. J. Mass Spectrom. 2000, 35, 773-783.

15. Ikonomou, M. G.; Kebarle, P. A Heated Electrospray Source for MassSpectrometry of Analytes from Aqueous Solutions. J. Am. Soc. Mass Spectrom. 1994, 5, 791-799. 